\title{
Partial Differential Equation With a Numerical Solution
}

\section{Haydir Ali Hassan}

Department of Mathematics, College of Education, University of Mustansiriyah Hydirali65@gmail.com

\section{Abstract}

The aim of this paper is to solve the linear partial differential equation numerically by using explicit optimal time method, an example is solved to show the effectiveness of the method..

\section{Introduction:}

Giventhe partial differential equation

$\frac{\partial z}{\partial t}=\frac{\partial^{2} z}{\partial x^{2}}-t x \frac{\partial z}{\partial x}-t z+f(x, t)$

Where $\mathrm{z}$ depending on $x$ and $t$

In this paper partial differential equation (1) will be so solved numerically by using explicit optimal time method.

By central difference we have $\frac{\partial^{2} z}{\partial x^{2}}=\frac{\partial}{\partial x}\left(\frac{\partial z}{\partial x}\right)=\frac{\partial}{\partial x}\left(\frac{z_{i+1}-z_{i}}{h}\right)$, and if we let $w=\frac{z_{i+1}-z_{i}}{h}$ then, $\frac{\partial w}{\partial x}=\frac{z_{i}-z_{i-1}}{h}$, and also we have

$\frac{\partial^{2} z}{\partial x^{2}}=\frac{z_{i+1}-2 z_{i}+z_{i-1}}{h^{2}}$

From (1)

$t x \frac{\partial z}{\partial x}+t z=t x_{i} \frac{z_{i}-z_{i-1}}{h}+t z_{i}$

Where $i=0,1, \ldots, N$ and $N$ is the partition of the interval By substitution (2) and (3) in (1) we have

$\grave{u}\left(x_{i}, t\right)=\left(\frac{1}{h^{2}}+\frac{t x_{i}}{h}\right) z_{i-1}+\left(\frac{-2}{h^{2}}-\frac{t x_{i}}{h}-t\right) z_{i}+\frac{1}{h^{2}} z_{i+1}+f\left(x_{i}, t\right)$

$i=0,1,2, \ldots, N$

if we let $a=\frac{1}{h^{2}}+\frac{t x_{i}}{h}, b=\frac{-2}{h^{2}}-\frac{t x_{i}}{h}-t$, and $c=\frac{1}{h^{2}}$ then (4) can becomes

$\grave{z}\left(x_{i}, t\right)=a z_{i-1}+b z_{i}+c z_{i+1}+f\left(x_{i}, t\right)$

And for $i=0,1, \ldots, N$ we have the following system:

$\grave{z}\left(x_{0}, t\right)=b z_{0}+c z_{1}+f\left(x_{0}, t\right)$

$\grave{z}\left(x_{1}, t\right)=a z_{0}+b z_{1}+c z_{2}+f\left(x_{1}, t\right)$

.........

(........

........

$\grave{z}\left(x_{N-1}, t\right)=a z_{N-2}+b z_{N-1}+c z_{N}+f\left(x_{N-1}, t\right)$

$\grave{z}\left(x_{N}, t\right)=a z_{N-1}+b z_{N}+c z_{N+1}+f\left(x_{N}, t\right)$

By using matrix system the above system becomes 


$\left[\begin{array}{c}\grave{z}\left(x_{0}, t\right) \\ \grave{z}\left(x_{1}, t\right) \\ \cdot \\ : \\ \dot{z}\left(x_{N-1}, t\right) \\ \grave{z}\left(x_{N}, t\right)\end{array}\right]=\left[\begin{array}{ccccccc}b & c & 0 & \cdot & \cdot & \cdot & 0 \\ a & b & c & 0 & \cdot & \cdot & 0 \\ 0 & a & b & c & \cdot & : & 0 \\ \cdot & \cdot & \cdot & \cdot & \cdot & \cdot & \cdot \\ \cdot & \cdot & \cdot & \cdot & \cdot & \cdot & \cdot \\ \cdot & \cdot & \cdot & \cdot & \cdot & \cdot & \cdot \\ 0 & 0 & \cdot & \cdot & a & b & c \\ 0 & 0 & \cdot & \cdot & \cdot & a & b\end{array}\right]\left[\begin{array}{c}z\left(x_{0}, t\right) \\ z\left(x_{1}, t\right) \\ \cdot \\ \vdots \\ \cdot \\ z\left(x_{N-1}, t\right) \\ z\left(x_{N}, t\right)\end{array}\right]+\left[\begin{array}{c}f\left(x_{0}, t\right) \\ f\left(x_{1}, t\right) \\ \cdot \\ \vdots \\ \cdot \\ f\left(x_{N-1}, t\right) \\ f\left(x_{N}, t\right)\end{array}\right]$

System (6) can be written as follows:

$\frac{d z}{d t}=G(t) z(t)+f(t)$

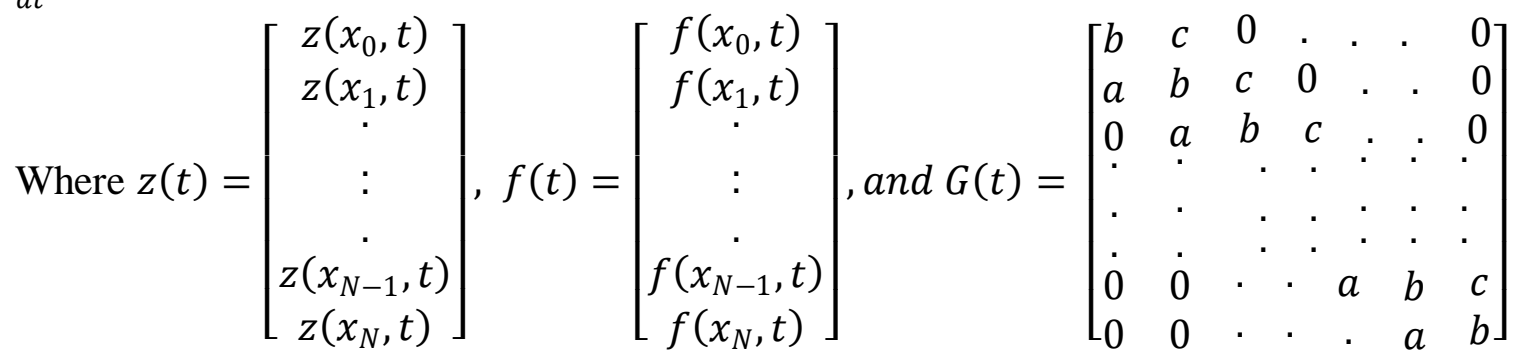

By using the value of $a, b$, and $c$ we have:

$$
G(t)=\left[\begin{array}{cccccc}
\frac{-2}{h^{2}}-\frac{t x_{0}}{h}-t & \frac{1}{h^{2}} & 0 & 0 & 0 & 0 \\
\frac{1}{h^{2}}+\frac{t x_{1}}{h} & \frac{-2}{h^{2}}-\frac{t x_{1}}{h}-t & \frac{1}{h^{2}} & 0 & 1 & \\
0 & \frac{1}{h^{2}}+\frac{t x_{2}}{h} & \frac{-2}{h^{2}}-\frac{t x_{2}}{h}-t & \frac{1}{h^{2}} & & \\
\vdots & 0 & & \frac{1}{h^{2}}+\frac{t x_{N-1}}{h} \frac{-2}{h^{2}}-\frac{t x_{N-1}}{h}-t & \frac{1}{h^{2}} \\
0 & & & \frac{1}{h^{2}}+\frac{t x_{N}}{h} & \frac{-2}{h^{2}}-\frac{t x_{N}}{h}-t
\end{array}\right]
$$

Let $U=\frac{G\left(t_{j}\right)+G\left(t_{j+1}\right)}{2}$, then
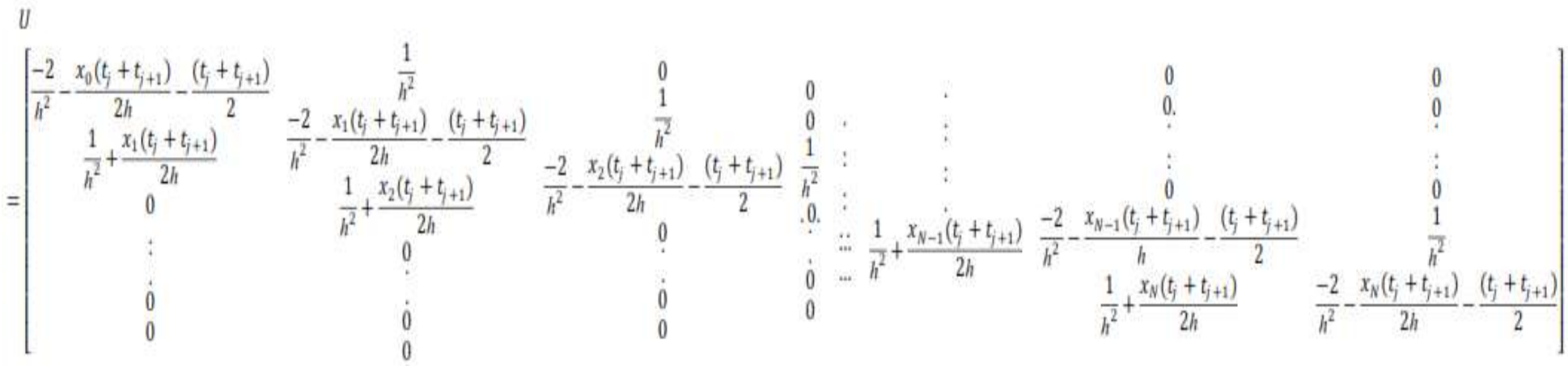

Where $j=0,1,2, \ldots, n$, with $\mathrm{n}$ the partition of the interval of $t$

If we set $\bar{a}=\frac{1}{h^{2}}+\frac{x_{1}\left(t_{j}+t_{j+1}\right)}{2 h}, \bar{b}=\frac{-2}{h^{2}}-\frac{x_{0}\left(t_{j}+t_{j+1}\right)}{2 h}-\frac{\left(t_{j}+t_{j+1}\right)}{2}$, and $\bar{c}=\frac{1}{h^{2}}$, then 


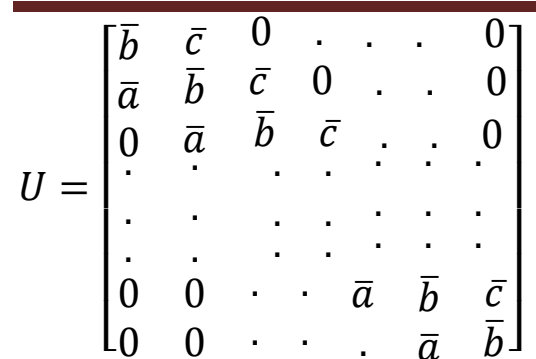

And hence the steeps to solve (7) is as follows:

1. Determine $h$ and $z\left(x_{0}\right)$

2. compute the matrix $U$

3. compute $z_{i+1}\left(t_{j}\right)=z_{i}\left(t_{j}\right)+\frac{\Delta t}{2}\left(U\left(t_{j}\right) z_{i}\left(t_{j}\right)+f_{i}\left(t_{j}\right)\right), i=0,1, \ldots, N-1$, and $j=$ $0,1, \ldots, n$

$$
z_{i+1}\left(t_{n+1}\right)=z_{i}\left(t_{n}\right)+\Delta t\left(U\left(t_{n}\right) z_{i}\left(t_{n}\right)+\frac{f_{i}\left(t_{n}\right)+f_{i}\left(t_{n}+\Delta t\right)}{2}\right.
$$

Numerical Example:

In this section the following numerical example will be solved to show the effectiveness of the method:

$\frac{\partial z}{\partial t}=\frac{\partial^{2} z}{\partial x^{2}}-t z-t x \frac{\partial z}{\partial x}+(t x-1) e^{-1} \cos (x-t)+e^{-t} \sin (x-t)$

$\mathrm{N}=5, \Delta t=0.1, z(0, t)=z(2 \pi, t)$, and $z(x, 0)=\sin x, h=\frac{2 \pi}{N}$, and $0 \leq t \leq 1$

Solution:

$\bar{a}=0.6333+\frac{x_{i}\left(t_{j}+t_{j+1}\right)}{2.5133}, \bar{b}=-1.2665-\frac{x_{i}\left(t_{j}+t_{j+1}\right)}{2.5133}-\frac{\left(t_{j}+t_{j+1}\right)}{2}, \bar{c}=0.6333$

The numerical results is given in the following table

\begin{tabular}{|c|c|c|c|c|c|c|}
\hline & $x_{0}=0$ & $x_{1}=\frac{2 \pi}{5}$ & $x_{2}=\frac{4 \pi}{5}$ & $x_{3}=\frac{6 \pi}{5}$ & $x_{4}=\frac{8 \pi}{5}$ & $x_{5}=2 \pi$ \\
\hline 0 & 0 & 0.94 & 0.5677 & -0.5677 & -0.94 & 0.0004 \\
\hline 0.1 & -0.0944 & 0.02126 & -0.0003 & 0.0713 & -0.261 & -0.08 \\
\hline 0.2 & -0.17 & -0.718 & 1.3 & -1.4 & 1.004 & -0.172 \\
\hline 0.3 & -0.22 & -1.71 & 2.8 & -2.95 & 2.0465 & -0.23 \\
\hline 0.4 & -0.2443 & -2.659 & 3.896 & -3.880 & 2.2764 & -0.2445 \\
\hline 0.5 & -0.2452 & -3.387 & 4.3622 & -3.9226 & 1.577 & -0.2451 \\
\hline 0.6 & -0.2336 & -3.7735 & 4.111 & -3.2019 & 0.19558 & -0.2334 \\
\hline 0.7 & -0.2095 & -3.802 & 3.2954 & -2.0238 & -1.4887 & -0.2093 \\
\hline 0.8 & -0.181 & -3.5333 & 2.1777 & -0.73552 & -3.410 & -0.1818 \\
\hline 0.9 & -0.1518 & -3.0344 & 1.0095 & 0.418 & -4.3637 & -0.1516 \\
\hline 1 & -0.1255 & -2.4353 & -0.02606 & 1.339 & -5.338 & -0.1253 \\
\hline
\end{tabular}

Table 1

From the above table we can see that, the first row is satisfy the condition $z(x, 0)=\sin x$, more over also we can see that the first column is very closely to the last column that is the condition $z(0, t)=z(2 \pi, t)$ is also satisfy. 


\section{References}

1. Said abdulmuti Al-Badawi, 1980. Advance Mathematics for Engineering. Third edition . Dar Al-ratibAljamiayahBirut

2. Ross, Shepley L. 1984. Differential Equations. Third edition. New Yourk: John Wiley \& Sons. Inc

3. Yu, Jui-Ling, 2007. An optimal Adaptive time-stepping scheme for solving ReactionDiffusion - chemotaxis systems. Mathematical Biosciences And Engineering 